\title{
Femtosecond electron diffraction: Preparation and characterization of (110)-oriented bismuth films
}

\section{$\operatorname{AUTHOR}(\mathrm{S})$ :}

Moriena, Gustavo; Hada, Masaki; Sciaini, German; Matsuo, Jiro; Miller, R. J. Dwayne

\section{CITATION:}

Moriena, Gustavo ...[et al]. Femtosecond electron diffraction: Preparation and characterization of (110)-oriented bismuth films. JOURNAL OF APPLIED PHYSICS 2012, 111(4): 043504.

\section{ISSUE DATE:}

2012-02-15

URL:

http://hdl.handle.net/2433/160655

\section{RIGHT:}

Copyright 2012 American Institute of Physics. This article may be downloaded for personal use only. Any other use requires prior permission of the author and the American Institute of Physics. The following article appeared in JOURNAL OF APPLIED PHYSICS 111, 043504 (2012) and may be found at http://link.aip.org/link/?jap/111/043504 


\section{AIP $\mid$ Appolied Physics}

\section{Femtosecond electron diffraction: Preparation and characterization of (110)-oriented bismuth films}

Gustavo Moriena, Masaki Hada, Germán Sciaini, Jiro Matsuo, and R. J. Dwayne Miller

Citation: J. Appl. Phys. 111, 043504 (2012); doi: 10.1063/1.3684975

View online: http://dx.doi.org/10.1063/1.3684975

View Table of Contents: http://jap.aip.org/resource/1/JAPIAU/v111/i4

Published by the American Institute of Physics.

\section{Related Articles}

Magnetostriction and $\Delta \mathrm{E}$ effect of melt-spun (Fe81-xCoxGa19)80B20 ribbons

J. Appl. Phys. 112, 053904 (2012)

Prevention of dewetting during annealing of FePt films for bit patterned media applications

Appl. Phys. Lett. 101, 092402 (2012)

Tailoring plasmon resonances in the deep-ultraviolet by size-tunable fabrication of aluminum nanostructures Appl. Phys. Lett. 101, 081110 (2012)

Nonadiabatic generation of coherent phonons

J. Chem. Phys. 137, 22A527 (2012)

Laser induced thermal-wave fields in multi-layered spherical solids based on Green function method

J. Appl. Phys. 112, 033521 (2012)

\section{Additional information on J. Appl. Phys.}

Journal Homepage: http://jap.aip.org/

Journal Information: http://jap.aip.org/about/about_the_journal

Top downloads: http://jap.aip.org/features/most_downloaded

Information for Authors: http://jap.aip.org/authors

\section{ADVERTISEMENT}

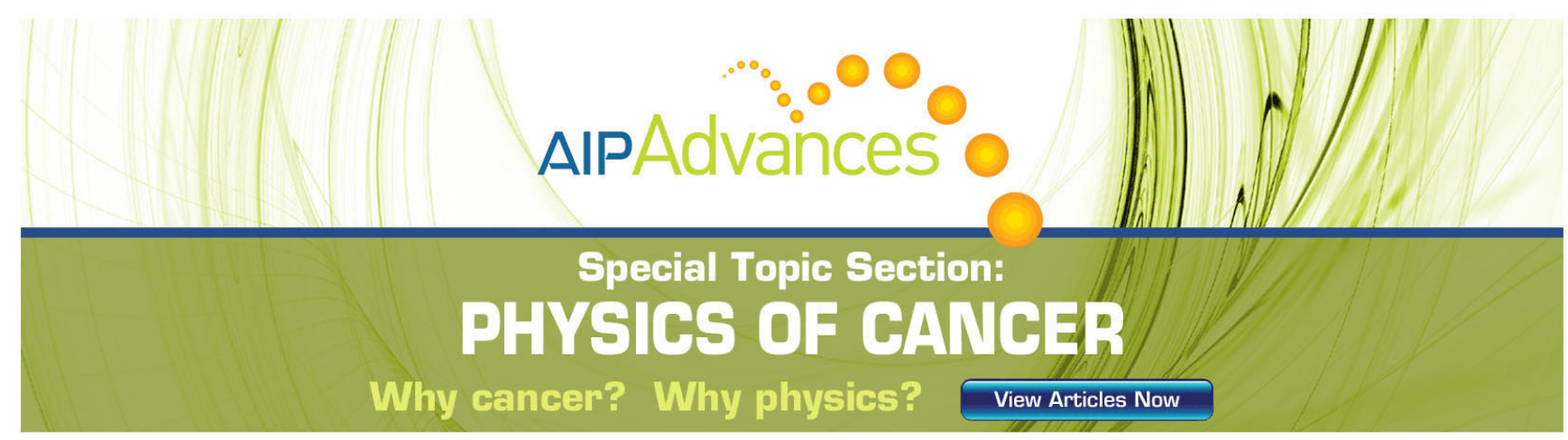




\title{
Femtosecond electron diffraction: Preparation and characterization of (110)-oriented bismuth films
}

\author{
Gustavo Moriena, ${ }^{1}$ Masaki Hada, ${ }^{2,3}$ Germán Sciaini, ${ }^{1,2}$ Jiro Matsuo, ${ }^{3, a)}$ \\ and R. J. Dwayne Miller ${ }^{1,2, b)}$ \\ ${ }^{1}$ Department of Chemistry and Physics, University of Toronto, 80 St. George Street, Toronto, Ontario \\ M5S3H6, Canada \\ ${ }^{2}$ Max Planck Research Department for Structural Dynamics, University of Hamburg, Center for Free Electron \\ Laser Science, DESY, Notkestrasse 85, 22607 Hamburg, Germany \\ ${ }^{3}$ Quantum Science and Engineering Center, Kyoto University, Gokasho, Uji Kyoto 611-0011, Japan
}

(Received 4 November 2011; accepted 11 January 2012; published online 16 February 2012)

\begin{abstract}
Here, we present a new approach to synthesize (110)-oriented ultrathin membranes of bismuth (Bi). This rather exotic orientation was achieved by directing the growth through rationale control of lattice matching. Bi films were hetero-epitaxially grown on the (100)-surface of freshly cleaved potassium chloride crystals. The sample orientation was characterized by $\mathrm{x}$-ray and electron diffraction. In addition, high quality free-standing films were obtained after dissolution of the substrate in water and controlled evaporation. Femtosecond electron diffraction (FED) was, therefore, used to monitor the coherent shear acoustic phonons in (110)-oriented free-standing Bi films produced by impulsive femtosecond optical excitation. The small de Broglie wavelength (flat Ewald sphere) of keV-electrons combined with an off-Bragg detection scheme provided a magnified view of shear atomic motions, i.e., lattice distortions in the transverse direction. Alloptical pump-probe experiments are usually insensitive to shear displacements, a fact that makes FED a unique non-contact method to achieve the complete characterization of elastic properties of nanoscale materials. (C) 2012 American Institute of Physics. [doi:10.1063/1.3684975]
\end{abstract}

\section{INTRODUCTION}

The determination of the elastic properties of nanoscale objects is of key importance for the design of nano-devices. For such small objects, contact methods are difficult to implement and noncontact all-optical spectroscopies are usually insensitive to shear modes whose signal is typically within the noise level in conventional pump-probe experiments. In such experiments a short optical pulse excites the sample to generate thermal stress. The stress is released as strain waves that modulate the optical properties of the film which are probed by a second optical pulse. When a thin free-standing film is considered, the film's surfaces establish boundary conditions for the formation of standing waves. The frequencies $\left(f_{n}\right)$ that fulfill the standing wave condition are given by

$$
f_{n}=\frac{n v_{s}}{2 d},
$$

where $v_{s}$ is the speed of sound along a given direction, $d$ is the film thickness, and $n$ is an integer number characterizing the different modes. Recently, femtosecond optical pumpprobe experiments performed on free-standing Si membranes with a thickness of a few hundred nanometers were able to detect up to the 19th harmonic order of the longitudinal acoustic mode. ${ }^{1}$ The determination of transverse acoustic waves by optical means is, however, much more difficult to implement. Transverse acoustic waves have been detected in GaAs and Zn single crystals film using a $p$-polarized beam at

\footnotetext{
${ }^{\text {a)} E l e c t r o n i c ~ m a i l: ~ m a t s u o . j i r o .7 s @ n u c l e n g . k y o t o-u . a c . j p . ~}$

${ }^{b)}$ Electronic mail: dwayne.miller@mpsd.cfel.de.
}

oblique angles of incidence. ${ }^{2}$ Shear acoustic waves have been also detected in aluminum exciting with a $\sim 1 \mu$ m laser spot. ${ }^{3}$ Using multiple excitation pulses in particular configurations, other mechanism such as stimulated Brillouin scattering can be exploited for the generation of transverse acoustic modes. ${ }^{4}$ In contrast, direct methods to detect the coherent vibrations in thin films have been reported using ultrashort electron or x-ray probe pulses which are capable of probing the atomic structure. Several dynamical studies were performed by photoexciting thin films and detecting coherent acoustic phonons using time-resolved $\mathrm{x}-\mathrm{ray}^{5-8}$ and electron diffraction techniques. ${ }^{9,10}$ Very recently, coherent acoustic phonons with higher frequencies than that predicted from Eq. (1) (with $n=1$ ) were detected in polycrystalline $\mathrm{Bi}$ films using electron diffraction probe. The origin of these coherent waves was discussed in terms of an optical-acoustic phonon decay. ${ }^{11}$

In this study, we demonstrate femtosecond electron diffraction (FED) to detect coherent shear waves in $30 \mathrm{~nm}$ thick, (110)-oriented free-standing bismuth (Bi) films. The small de Broglie wavelength for $\mathrm{keV}$-electrons results in a flat Ewald sphere that in combination with off Bragg detection provides a magnified view of shear motions. This unique feature to the diffraction process makes FED an ideal non-contact method for the study of elastic properties of nanomaterials. ${ }^{10}$

\section{SAMPLE PREPARATION AND CHARACTERIZATION OF (110)-ORIENTED BISMUTH}

The additional motivation for this work is that $\mathrm{Bi}$ is a Peierls distorted face centered cubic lattice. One would 
ideally use (110) orientated crystals in order to directly observe the collective photoinduced bond softening atomic motions along the (111) plane in diffraction. ${ }^{12}$ The preparation of (110)-oriented Bi films is a difficult task. ${ }^{13} \mathrm{Bi}$ usually crystallizes in the (111) orientation on a variety of substrates such as silicon, sapphire, etc. ${ }^{14,15}$ In this study we were able to prepare (110)-highly oriented $\mathrm{Bi}$ films by heteroepitaxially growing $\mathrm{Bi}$ on the (100) surface of freshly cleaved potassium chloride $(\mathrm{KCl})$ crystals. The quality and orientation of the Bi film strongly depend on the quality of the underlying substrate. The unit cell of $\mathrm{Bi}$ is rhombohedral and resides in the space group of R3(-)m (No. 166). ${ }^{16}$ The lattice parameters $a$ and $\alpha$ are $0.4746 \mathrm{~nm}$ and $57.24^{\circ}$, respectively. The [111] zone axis is perpendicular to (111) plane. Figure 1(a) shows a schematic of the Bi (111) plane on a sapphire (0001) plane. The interatomic distance of $0.275 \mathrm{~nm}$ on the (0001) surface of sapphire is in close proximity to that of the $0.263 \mathrm{~nm}\left(\frac{2 \sqrt{3}}{3} a \sin (\alpha / 2)\right)$ projection of the (111) plane of $\mathrm{Bi}$. The value of misfit is, therefore, $4.6 \%$. As an additional consideration, in order to study the intrinsic properties of $\mathrm{Bi}$ on the nanoscale, the sapphire substrate strongly perturbs the boundary conditions. The ideal system is a free standing nanofilm. In order to accomplish this, the nanofilm has to be grown on a substrate that can be easily removed from. In this respect, $(100)-\mathrm{KCl}$ or sodium chloride $(\mathrm{NaCl})$ surfaces provide ideal solutions as $\mathrm{Bi}$ can be grown hetero-epitaxially along the [11-1] zone axis which is perpendicular to the (110) plane. Figure 1(b) shows a schematic of the lattice matching between the (110) plane of Bi and the (100) plane of $\mathrm{KCl}$. As shown in the figure, the nearest neighbor atom distances $\mathrm{K}^{+}-\mathrm{K}^{+}$or $\mathrm{Cl}^{-}-\mathrm{Cl}^{-}(0.4449 \mathrm{~nm})$, matches very well both, the lattice parameters $a(0.4746 \mathrm{~nm})$ and $2 a \sin (\alpha / 2)(0.4547 \mathrm{~nm})$ of $\mathrm{Bi}$. The value of misfit is in this case $6.2 \%$ for $a$ and $2.1 \%$ for $2 a \sin (\alpha / 2)$.

In order to perform the vapor deposition, $\mathrm{KCl}$ crystals cleaved along the (100) plane were transferred to a vacuum chamber. The chamber was evacuated to $4 \times 10^{-5} \mathrm{~Pa}$ with a cryogenic pump and high purity Bi (99.99\%) was heated up in a tungsten boat. The deposition rate was $0.1 \mathrm{~nm} / \mathrm{s}$ which guarantees a homogeneous film thickness. The thickness was constantly monitored with a quartz oscillator $(6 \mathrm{MHz}$; INFICON) and determined to be $30 \pm 1 \mathrm{~nm}$. Bi films were released by controllably immersing the substrates in water. The floating Bi films were then transferred to a host supporting Si mesh and dried in a controlled environment.

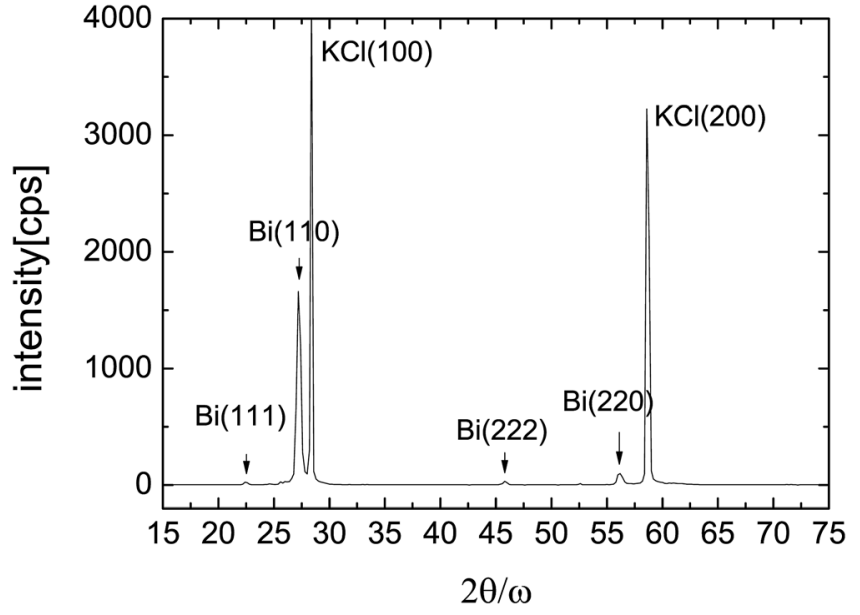

FIG. 2. Typical XRD from the $\mathrm{Bi} / \mathrm{KCl}$ sample.

The crystallinity of the $\mathrm{Bi}$ film deposited on $\mathrm{KCl}$ was characterized using $\mathrm{x}$-ray diffraction (XRD) measurements with $\mathrm{Cu} \mathrm{K} \alpha$ X-ray radiation $(\lambda=0.154 \mathrm{~nm})$ on a computercontrolled X-ray diffractometer (Rigaku, ATX-G, $18 \mathrm{~kW}$ ). Figure 2 shows the typical XRD obtained from the Bi film where the $\mathrm{K} \alpha \mathrm{XRD}$ lines from $(110)$ plane $\left(2 \theta=27.162^{\circ}\right)$ and $(220)$ plane $\left(2 \theta=56.021^{\circ}\right)$ are shown. In the same XRD, small peaks of $\mathrm{K} \alpha \mathrm{X}$-ray diffraction lines from $\mathrm{Bi}$ (111) plane $\left(2 \theta=22.468^{\circ}\right)$ and $\mathrm{Bi}(222)$ plane $\left(2 \theta=45.863^{\circ}\right)$ can be also observed. The $\mathrm{Bi}(110) / \mathrm{Bi}$ (111) integral intensity ratio for powder $\mathrm{Bi}$ (random orientation) is $\sim 31.3$ as obtained by using CRYSTALMAKER 8.2. ${ }^{17}$ In the case of our sample we obtained a value of $\sim 149.8$ for the same ratio. The degree of orientation (110) relative to (111) estimated from the relative intensities was found to be 83:17.

The experimental FED setup used in this work has been described elsewhere. ${ }^{10}$ In the present study, 180-fs optical pulses with a carrier wavelength of $775 \mathrm{~nm}$ were used to excite the sample (with laser spot size of about $500 \mu \mathrm{m}$ ). The structural changes were monitored using $\sim 3 \times 10^{4} 50-\mathrm{keV}$ electrons in bunches of $<1 \mathrm{ps}$ in duration ${ }^{18}$ (with a lateral spot size of $150 \mu \mathrm{m}$ ). Figure 3(a) shows a typical diffraction pattern obtained from (110)-oriented $\mathrm{Bi}$. In contrast to the typical 12-fold symmetry shown by (111)-oriented $\mathrm{Bi}$ films,${ }^{14,15}$ a clear diffraction pattern with fourfold symmetry was observed. The diffraction pattern agreed with the simulation performed (Fig. 3(b)) using CRYSTALMAKER 8.2, ${ }^{17}$ (a)

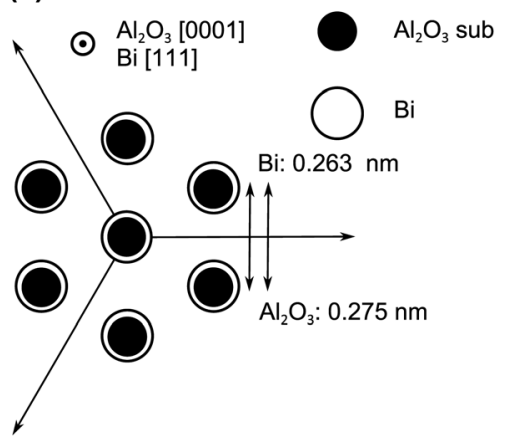

(b)

$$
\odot \underset{\mathrm{Bi}[11 \overline{1}]}{\mathrm{KCl}[001]}
$$

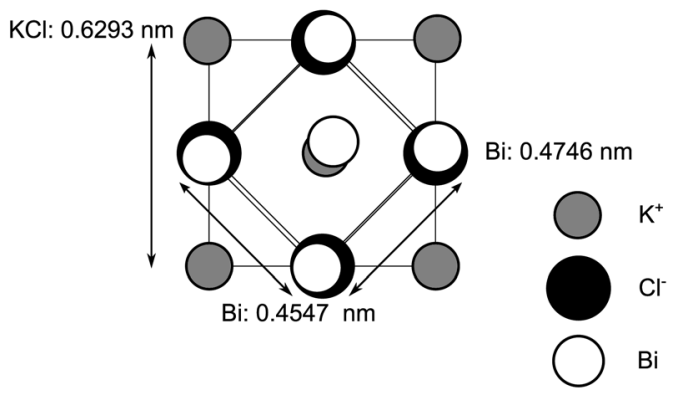

FIG. 1. (a) Schematic of orientation of Bi (111) plane on sapphire (0001) plane, and (b) $\mathrm{Bi}$ (110) plane on $\mathrm{KCl}(100)$ plane. 

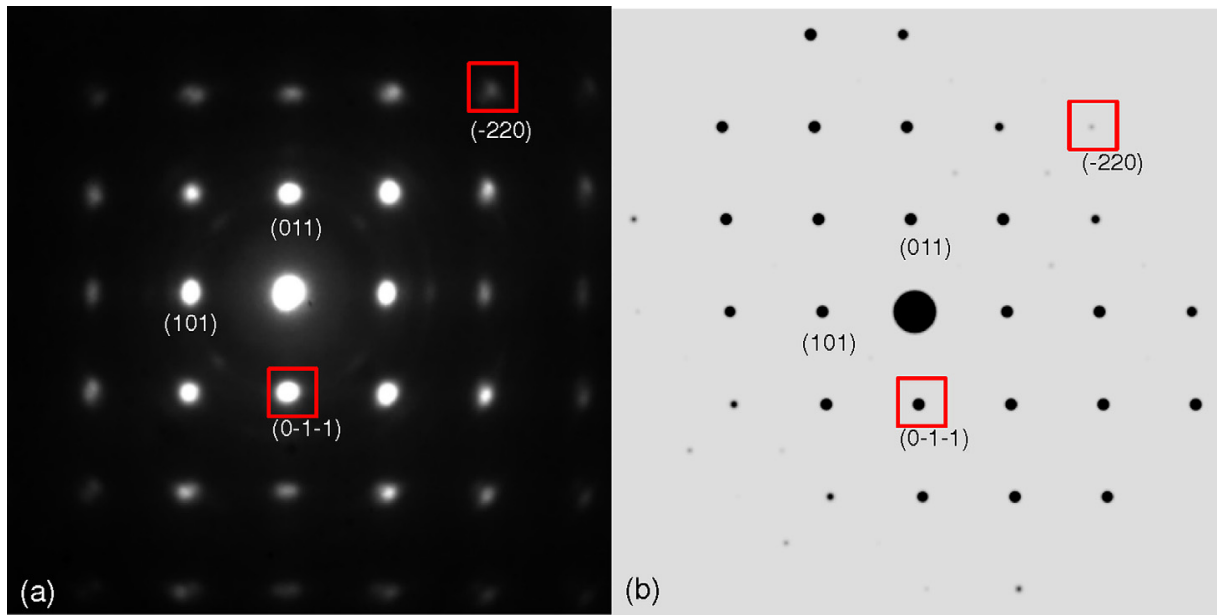

FIG. 3. (Color online) (a) Diffraction pattern from $\mathrm{Bi}(110)$ with FED measurements, and (b) computer simulated diffraction pattern from Bi (110). The diffraction indexes are inserted in the figure.

for an electron beam propagating along the [11-1] zone axis and corroborates the (110)-orientation of film. As shown in Fig. 4(a), the first order diffraction spots correspond to the (101) and (011) Bragg reflections.

\section{RESULTS AND DISCUSSION}

The film was photoexcited with a fluence below the damage threshold of $\sim 2 \mathrm{~mJ} / \mathrm{cm}^{2}$. The generation of shear acoustic modes is due to non-uniform heat deposition introduced by the Gaussian intensity profile of the pump pulse and associated surface deformation, as well as the periodic boundary condition imposed by the supporting grid structure. ${ }^{10}$ Diffraction patterns were acquired at time delays between $-38 \mathrm{ps}$ and $450 \mathrm{ps}$ with a time step of 2 ps. The integral intensities were calculated adding individual intensities of each pixel inside a square box around the peak of interest. Figure 4 shows the dynamics of the integrated intensities after normalization $\left(I(t) / I_{\mathrm{t}<\mathrm{t} 0}\right)$ and the peak indicated as $(-220)$ and $(0-1-1)$. Some interesting features can be found in these dynamics. Immediately after photoexcitation, there is a fast drop of $3-8 \%$ observed in the diffracted intensity. Following

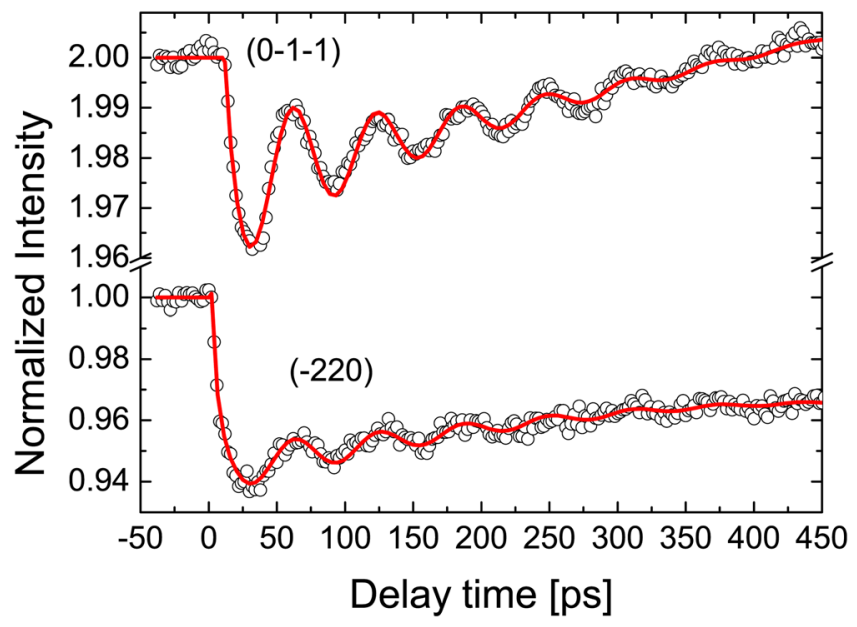

FIG. 4. (Color online) Kinetics of the electron diffraction from $(-220)$ and (0-1-1) for an excitation fluence of $\sim 0.9 \mathrm{~mJ} / \mathrm{cm}^{2}$. Open circles represent the experimental data and solid lines are the fitting function. that drop, the diffracted intensity shows a damped oscillatory behavior. Apart from this, no other important changes in the average intensity can be observed over the explored delay time (see the kinetics of the electron diffraction from $(-220))$. This last feature is an indication that no appreciable heat transport out of the excited region occurs in the temporal window of the experiment. The typical time to cool the film back down to the initial temperature ${ }^{10}$ is several orders of magnitude longer than the maximum time explored in this experiment, estimated to be in the $\mu$ s time scale. ${ }^{10}$

Because of the complexity of the dynamics of these peaks, a phenomenological function that takes into consideration the fast drop of the diffraction intensity and damped oscillations was used to fit the signal after photoexication $\left(t>t_{0}\right)$. An extra term to consider the baseline behavior was also included.

$$
\begin{aligned}
I(t)= & A_{1}+\left(1-A_{1}\right) \exp \left(-\left(t-t_{0}\right) / \tau_{1}\right) \\
& +A_{2} \sin \left(2 \pi f_{1} t+\phi\right) \exp \left(-\left(t-t_{0}\right) / \tau_{2}\right) \\
& +A_{3} \sin \left(2 \pi f_{2} t\right)
\end{aligned}
$$

The three important parameters extracted from this fitting function are: the initial fast decay time constant, $\tau_{1}$; the oscillation frequency, $f_{1}$; and, the damping time constant, $\tau_{2}$. The values of 2-4 ps obtained for $\tau_{1}$, are in good agreement with the characteristic time for electron-phonon energy transfer found by Sciaini et al. ${ }^{19}$ and Esmail et al. ${ }^{20}$ The values obtained for $f_{1}$ and $\tau_{2}$ are $16.3 \pm 0.5 \mathrm{GHz}$ and 100 to 200 ps for the 19 diffraction spots considered. Within the signal-to-noise level, only one main oscillation at the frequency of about $16.3 \mathrm{GHz}$ was observed. Solving Eq. (1) for $v_{s}$ and using the obtained values for the frequency, $f_{1}=16.3 \mathrm{GHz}{ }^{21}$ a sound velocity of $980 \pm 60 \mathrm{~m} / \mathrm{s}$ is obtained. This result is in agreement with the velocities $v_{13}=910 \pm 1 \mathrm{~m} / \mathrm{s} \quad v_{14}=1055 \pm 6 \mathrm{~m} / \mathrm{s}$ obtained by Eckstein et al. ${ }^{22}$ The velocities $v_{13}$ and $v_{14}$ correspond to the shear polarized modes propagating along the [11-1] direction. This fact indicates that the shear elasticity of $30 \mathrm{~nm}$ thick Bi films is in practice the same as bulk $\mathrm{Bi}$. The quality of the data in Fig. 4 to draw this conclusion is exceptional for such ultrathin sample, especially for a table top source, and would be difficult to attain with any other method. 


\section{CONCLUSION}

(110)-highly oriented Bi films were hetero-epitaxially grown on $\mathrm{KCl}$ (100) substrates. Coherent acoustic transverse standing waves in the tens of Gigahertz range were observed by FED. The velocity of the coherent acoustic shear mode was in agreement with those found for bulk Bi along the [11-1] direction. The acoustic modes are very sensitive to the density, impurities and other mechanical properties of nanoscale materials. Therefore, it is interesting to note that with both longitudinal and transverse elastic modes obtained directly via impulsive optical excitation, FED opens a new route toward the full characterization of elastic properties of nanoscale materials. It would be interesting to extend the present studies to thinner films in order to observe the onset of confining effects on the mechanical properties of the film as well as the onset of photoinduced phase transitions in which the shear mode collapses upon formation of the liquid state to directly observe the many body effects involved in this strongly coupled electron-lattice system.

\section{ACKNOWLEDGMENTS}

This research was supported by the Natural Science and Engineering Research Council of Canada and the Canada Foundation for Innovation and partially supported by the Core Research for Evolutional Science and Technology (CREST) program of the Japan Science and Technology Agency (JST).

${ }^{1}$ F. Hudert, A. Bruchhausen, D. Issenmann, O. Schecker, R. Waitz, A. Erbe, E. Scheer, T. Dekorsy, A. Mlayah, and J.-R. Huntzinger, Phys. Rev. B 79, 201307 (2009).

${ }^{2}$ O. Matsuda, O. B. Wright, D. H. Hurley, V. E. Gusev, and K. Shimizu, Phys. Rev. Lett. 93, 095501 (2004).
${ }^{3}$ C. Rossignol, J. M. Rampnoux, M. Perton, B. Audoin, and S. Dilhaire, Phys. Rev. Lett. 94, 166106 (2005).

${ }^{4}$ K. Nelson, J. Appl. Phys.53, 6060 (1982).

${ }^{5}$ U. Shymanovich, M. Nicoul, J. Blums, K. Sokolowski-tinten, A. Tarasevitch, T. Wietler, M. Horn von Hoegen, and D. von der Linde, Appl. Phys, A 87, 7 (2007).

${ }^{6}$ M. Bargheer, N. Zhavoronkov, Y. Gritsai, J.C. Woo, D.S. Kim, M. Woerner, and T. Elsaesser, Science 306, 1771 (2004).

${ }^{7}$ A. Cavalleri, C. W. Siders1, F. L. H. Brown, D. M. Leitner, C. Tóth, J. A. Squier, C. P. J. Barty, K. R. Wilson, K. Sokolowski-Tinten, M. H. von Hoegen, D. von der Linde, and M. Kammler, Phys. Rev. Lett. 85, 586 (2000).

${ }^{8}$ C. Rose-Petruck, R. Jimenez, T. Guo, A. Cavalleri, C. W. Siders, F. Raksi, J. A. Squier, B. C. Walker, K. R. Wilson, and C. P. J. Barty, Nature 398, 310 (1999).

${ }^{9}$ S. Nie, X. Wang, H. Park, R. Clinite, and J. Cao, Phys. Rev. Lett. 96, 025901 (2006).

${ }^{10}$ M. Harb, W. Peng, G. Sciaini, C. T. Hebeisen, R. Ernstorfer, M. A. Eriksson, M. G. Lagally, S. G. Kruglik, and R. J. D. Miller, Phys. Rev. B 79, 094301 (2009).

${ }^{11}$ A. Bugayev, A. Esmail, M. Abdel-Fattah, and H. E. Elsayed-Ali, AIP Adv. 1, 012117 (2011).

${ }^{12}$ K. Sokolowski-Tinten, C. Blome, J. Blums, A. Cavalleri, C. Dietrich, A. Tarasevitch, I. Uschmann, E. Forster, M. Kammler, M. H. von Hoegen, and D. von der Linde, Nature 422, 287 (2003).

${ }^{13}$ K. S. Wu and M. Y. Chern, J. Appl. Phys. 104, 033704 (2008).

${ }^{14}$ M. Kammler and M. H. von Hoegen, Surf. Sci. 576, 56 (2005).

${ }^{15}$ T. Payer, I. Rajkoviác, M. Ligges, D. von der Linde, M. H. von Hoegen, and F.-J. Meyer zu Heringdorf, Appl. Phys. Lett. 93, 093102 (2008).

${ }^{16}$ D. Schiferl and C. S. Barrett, J. Appl. Cryst. 2, 30 (1969).

${ }^{17}$ CrystalMaker Software Limited, CRYSTALMAKER 8.2, http:// www.crystalmaker.com/.

${ }^{18}$ C. T. Hebeisen, G. Sciaini, M. Harb, R. Ernstorfer, T. Dartigalongue, S. G. Kruglik, and R. J. D. Miller, Opt. Express 16, 3334 (2008).

${ }^{19}$ G. Sciaini, M. Harb, S. G. Kruglik, T. Payer, C. T. Hebeisen, F.-J. Meyer zu Heringdorf, M. Yamaguchi, M. H. von Hoegen, R. Ernstorfer, and R. J. D. Miller, Nature 458, 56 (2009).

${ }^{20}$ A. R. Esmail and H. E. Elsayed-Ali, Appl. Phys. Lett. 99, 161905 (2011).

${ }^{21}$ M. Chergui, D. M. Jonas, E. Riedle, R. W. Schoenlein, and A. J. Taylor, Ultrafast Phenomena XVII (Oxford University Press, New York, 2010), p. 284.

${ }^{22}$ Y. Eckstein, A. W. Lawson, and D. H. Reneker, J. Appl. Phys. 31, 1534 (1960). 